\title{
Differences in genetic predisposition to Graves' disease (GD) and Graves' orbitopathy (GO) between young and elderly patients.
}

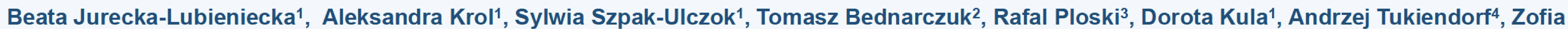 \\ Kolosza ${ }^{4}$, Barbara Jarzab ${ }^{1}$ \\ 1Department of Nuclear Medicine and Endocrine Oncology, Maria Sklodowska-Curie Memorial Cancer Center and Institute of Oncology, Gliwice Branch, Poland: \\ 2Department of Internal Medicine and Endocrinology, Medical University of Warsaw, Warsaw, Poland; \\ ${ }^{3}$ Department of Medical Genetics, Forensic Medicine, Medical University of Warsaw, Warsaw, Poland \\ ${ }^{4}$ Department of Epidemiology, Maria Sklodowska-Curie Memorial Cancer Center and Institute of Oncology, Gliwice Branch, Poland;
}

\section{Introduction}

Graves' disease is a complex disorder. Genetic predisposition modified by environmental factors is responsible for the pathogenesis of GD. Young patients are susceptible to environmental factors for a shorter time than older patients, thus the impact of genetic factors may be higher than environmental factors in patients with young age of GD diagnosis. Differences in the disease phenotype between younger and older patients may suggest a different genetic predisposition to the GD. The association between genetic markers and the age at the diagnosis was reported in type I diabetes, rheumatoid arthritis and multiple sclerosis.

Orbitopathy (GO) and GD hyperthyroidism have the common autoantigen TSHR which plays a key role in triggering the onset of the disease. Taking into account the diverse nature of the $\mathrm{GO}$ phenotype, especially in young patients and lack of the relation of time of the $\mathrm{GO}$ with development of hyperthyroidism, the pathogenesis of GD and GO may correlate with different genetic backgrounds. Current studies suggest a correlation between polymorphism in cytokines involved in orbit inflammation and GO, but not GD. It has been confirmed that GO risk increases with age and is considerably higher in smokers.

The aim of the study was to asses genetic predisposition to GD and $\mathbf{G O}$ in young patients (age of diagnosis $\leq \mathbf{3 0}$ years of age) in which the time of environmental effects was shorter than in older patients.

\section{Methods}

735 GD patients and 1216 healthy controls from Poland were included in the study. 338 of the patients had orbitopathy NOSPECS $\geq 2$. Association analyses were performed between genetic variants in genes encoding proteins involved in immunoresponse (HLADRB1, TNF, CTLA4, CD40, NFKb, PTPN22, IL4 and IL10), RTSH and the age of diagnosis of $G D$ and $G O$. Patients were stratified by the age of diagnosis of $\mathrm{GD}$ and $\mathrm{GO}$.

\section{Results}

Our analysis demonstrated an association between HLA DRB $01 * 03$ and the age of GD diagnosis. Patients with age $\leq 30$ years at GD diagnosis had higher frequency of the HLA DRB $1 * 03$ allele. The genotype containing at least one DRB $1^{*} 03$ allele occurred almost twice as often in younger patients (Tab. 1). Young carriers of the DR3 polymorphism were more common then the older ones both in familial and sporadic GD. This results suggest the existence of different genetic conditions for the development of GD in young people.

No association was observed between DRB1*03 allele and the duration of pharmacotherapy, GD relapse rate, number of radioiodine treatment courses, thyroid receptor (TR) Ab level and gender.

Tab. 1. HLA DRB1*03 genotypes in patients with GD stratified by the age at $\mathrm{GD}$ diagnosis.

\begin{tabular}{|c|c|c|c|c|}
\hline SNP & Genotype & $G D \leq 30 \mathrm{n}(\%)$ & GD >30 n (\%) & OR $(95 \% \mathrm{Cl}) ; \mathrm{p}$ \\
\hline \multirow{4}{*}{$\begin{array}{l}\text { HLA- } \\
\text { DRB1*03 }\end{array}$} & DR3- & $42(42)$ & $196(61)$ & Reference \\
\hline & DR3-/t+ & $56(55)$ & $118(37)$ & $2.21(1.36-3.31) ; p<0.00$ \\
\hline & DR3+/+ & 3 (3) & $7(2)$ & $2.00(0.31-9.18) ; \mathrm{ns}$ \\
\hline & DR3 $-/+$ and $+/+*$ & $59(58)$ & $125(39)$ & $2.20(1.36-3.57) ; p<0.001$ \\
\hline
\end{tabular}

We found a significant association between orbitopathy and HLA DRB $1{ }^{*} 03$ and TSHR gene rs 179247 polymorphisms.

We observed a higher frequency of the HLA DRB1 ${ }^{*} 03$ allele in young patients with $\mathrm{GO}(p<0.01)($ Tab. 2$)$

\section{Bibliography}

1. Jurecka-Lubieniecka B., Bednarczuk T., Ploski R., Krajewska J., Kula D., Kowalska M., Tukiendorf A., Kolosza Z., Jarzab B. Differences in gene-gene interactions in Graves' disease patients stratified by age of onset. PLoS One 2016, 4,11(3), $e 0150307$. 2. Jurecka-Lubieniecka B., Ploski R., Kula D., Szymanski K., Beanarczuk T., Ambroziak U., Hasse-Lazar K., Hyla-Klekot L., Tukiendorf A.,
Tab. 2. Distribution of HLA DRB $1 * 03$ polymorphism in GD patients stratified by the age of diagnosis of $\mathrm{GO}$.

\begin{tabular}{|c|c|c|c|c|c|}
\hline SNP & Genotype & NOSPECS & GD $\leq 30 \mathrm{n}(\%)$ & GD $>30 \mathrm{n}(\%)$ & $\mathbf{p}$ \\
\hline HLA DRB1*03 & DR32/2 & $2+$ & $15(35.7)$ & $112(63.6)$ & 0.001 \\
\hline & DR32/+ and +/+ & & $27(64.3)$ & $64(36.4)$ & \\
\hline & DR32/2 & $0-1$ & $26(44.8)$ & $84(57.9)$ & Ns 0.062 \\
\hline & DR32/+ and +/+* & & $32(55.2)$ & $61(42.1)$ & \\
\hline
\end{tabular}

In younger patients without orbitopathy the A allele of TRSH gene rs 179247 occurred significantly more often in comparison with those with GO $(p<0.013)$. In younger patients group there was a statistically significant difference in genotype distribution $(p<0.038)$. The presence of an AA homozygous locus was associated with a significant reduction in the risk of GO incidence as compared to patients with $A G$ or $G G$ genotypes $(p<0.019)($ Tab. 3)

Tab. 3. Distribution of rs179247 TSHR alleles and genotypes in GD patients with and without GO

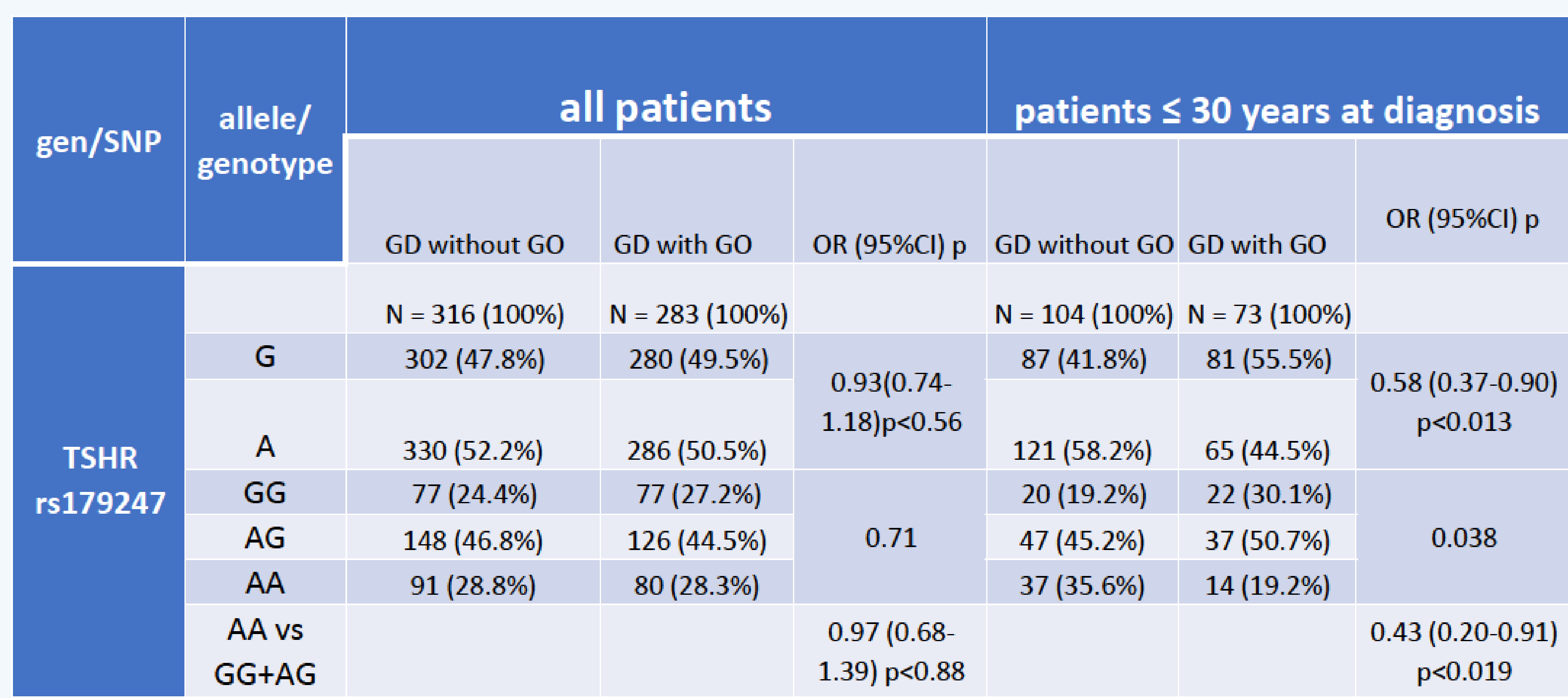

In our study the allele A carriers were dominant in the group of patients with orbitopathy $(\mathrm{N}=145$ vs $\mathrm{N}=90)$, especially in young patients group $(\mathrm{N}=64$ vs $\mathrm{N}=28$ ). Orbitopathy was not present in $86 \%$ of young $A A$ homozygote carriers $(\mathrm{N}=30$ vs $\mathrm{N}=5)$ (Fig. 1)

These findings showed the association between the presence of the allele A and lesser risk of GO in young GD patients

Fig. 1. Incidence of $\mathrm{GO}$ in patients with age at $\mathrm{GD}$ diagnosis $\leq 30$ years and with the AA genotype TSHR rs 179247
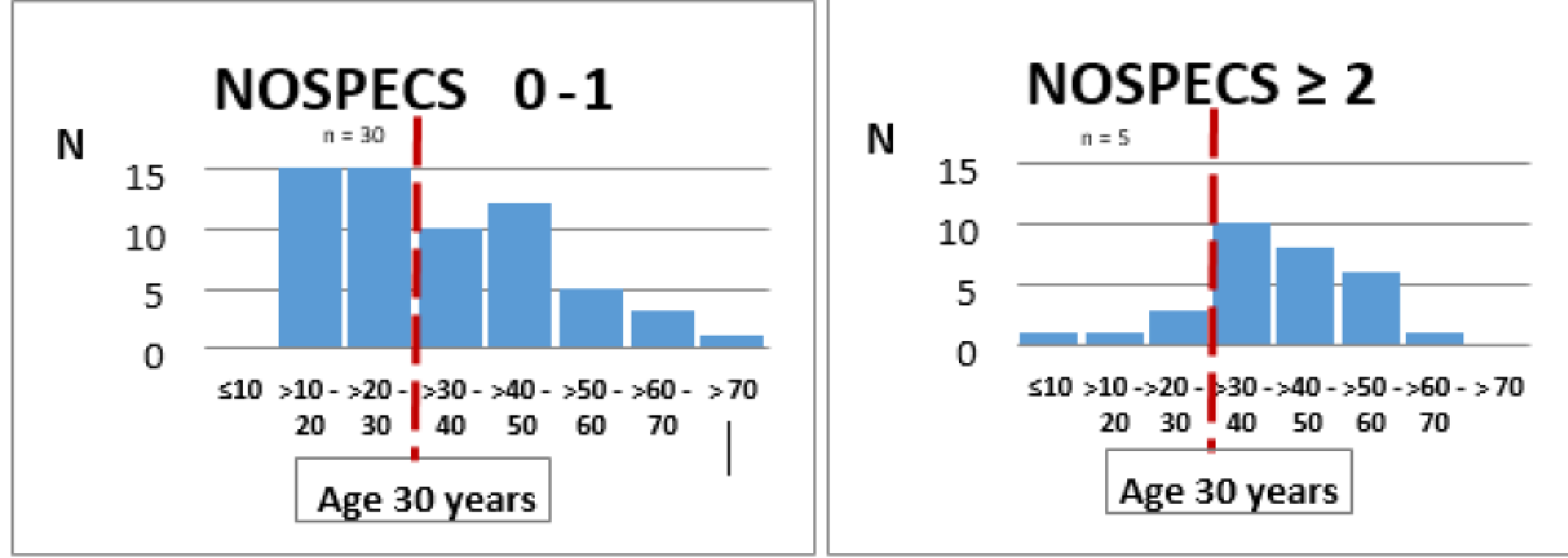

In the group of all patients or only older patients (age at diagnosis $>30$ years) the frequency of alleles present and genotype distributions of polymorphisms HLA DRB1 and TSHR rs179247 did not differ in patients with or without GO.

Conclusions

1. Polymorphism of HLA DRB $1{ }^{*} 03$ is associated with an early age at diagnosis of GD.

2. Carriers of the HLA DRB $1^{*} 03$ allele are significantly more common in young patients with $\mathrm{GO}$

3. The allele A of the rs179247 polymorphism in the TSHR gene is associated with a lower risk of GO in young patients with GD.

3. Jurecka-Lubieniecka B, Plosk R. Kula D. KrolA., Bednarczuk T., Kolosza Z, Tukiendorf A, Szpak-Ulczok S, StanjekCichoracka A., Polanska J., Jarzab B. Association between age at diagnosis of Graves' disease and variants in genes involved in immune response. PLOS One, 2013, 8, 3, e59349. 4. Ploski R., Brand O. J., JureckaLLublecka B., Franaszczyk M., Kula D., Krajewski P., Karamat M. A., Simmonds M. J. Franklyn J. A., Gough S. C., Jarzab B., Bednarczuk T. Thyrod st

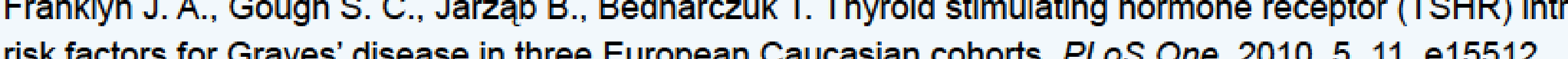

